\title{
Analysis of Water and Hydrogen Bond Dynamics at the Surface of an Antifreeze Protein
}

\author{
Yao Xu, Ramachandran Gnanasekaran, and David M. Leitner \\ Department of Chemistry and Chemical Physics Program, University of Nevada, Reno, NV 89557, USA \\ Correspondence should be addressed to David M. Leitner, dml@unr.edu
}

Received 14 December 2011; Accepted 7 March 2012

Academic Editor: Keli Han

Copyright () 2012 Yao Xu et al. This is an open access article distributed under the Creative Commons Attribution License, which permits unrestricted use, distribution, and reproduction in any medium, provided the original work is properly cited.

\begin{abstract}
We examine dynamics of water molecules and hydrogen bonds at the water-protein interface of the wild-type antifreeze protein from spruce budworm Choristoneura fumiferana and a mutant that is not antifreeze active by all-atom molecular dynamics simulations. Water dynamics in the hydration layer around the protein is analyzed by calculation of velocity autocorrelation functions and their power spectra, and hydrogen bond time correlation functions are calculated for hydrogen bonds between water molecules and the protein. Both water and hydrogen bond dynamics from subpicosecond to hundred picosecond time scales are sensitive to location on the protein surface and appear correlated with protein function. In particular, hydrogen bond lifetimes are longest for water molecules hydrogen bonded to the ice-binding plane of the wild type, whereas hydrogen bond lifetimes between water and protein atoms on all three planes are similar for the mutant.
\end{abstract}

\section{Introduction}

While the complex dynamics of large biological molecules and the connection to function have fascinated physical scientists for some time, in more recent years researchers have turned their attention to the interface of biomolecules with water. Coupling of protein and water dynamics, for example, has been examined by molecular simulations [1-10] and a growing number of experimental probes [11-14], and a wide variety of dynamical time scales have been found $[15,16]$ due to the heterogeneity of protein-water interactions. One class of proteins for which protein-water interactions are critical to function is antifreeze proteins (AFPs). AFPs are widely distributed in certain plants, vertebrates, fungi, and bacteria to provide cells protection in cold environments [17-20] but the mechanism for antifreeze activity is still not well understood. In this paper we analyze by all-atom molecular dynamics (MD) simulations the dynamics of water molecules and hydrogen bonds at the protein-water interface of the AFP from the spruce budworm Choristoneura fumiferana and a mutant that has little antifreeze activity. We calculate velocity autocorrelation functions and their power spectra for water molecules around the protein and we compute hydrogen bond time correlation functions for bonds between the protein and water. We obtain distinct spectra for the water around different regions of the protein, which are affected by mutation. Moreover, we observe longer hydrogen bonding between water molecules and the icebinding plane of this AFP compared to other parts of the protein, a difference that nearly disappears with mutation, indicating a correlation between hydrogen bond lifetimes and activity of this AFP.

AFPs were first discovered in several Antarctic fish species [21], AFPs that have since been classified as Type I. The generally accepted mechanism for the Type I AFP is the adsorption-inhibition mechanism [22-24], which proposes that AFPs adsorb onto the preferred growth sites of an ice surface, thereby preventing new ice growth [25]. It was initially thought that ice and AFP interacted through hydrogen bonding [22]. However, when parts of the protein that were thought to facilitate this hydrogen bonding were mutated, the hypothesized decrease in antifreeze activity was not observed, and hydrophobic interactions were suggested instead [26]. MD simulations have been carried out to sort out the possible mechanisms [6], but there is still no consensus on which sites of the protein interact with ice, 


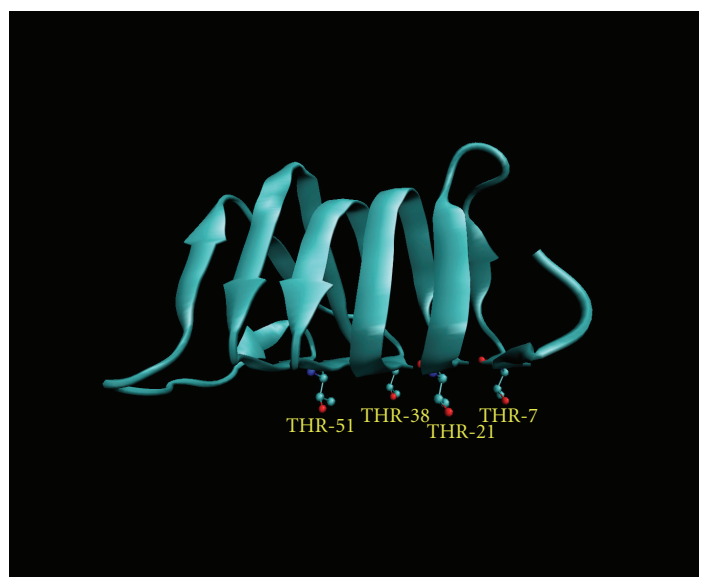

FIgURE 1: The structure of wild-type AFP from spruce budworm Choristoneura fumiferana, indicating the location of the four threonine residues on the ice-binding plane (Plane 1), which in our mutation studies we replace with leucine. Plane 2 is in the front and Plane 3 is in the back.

or whether the protein inhibits growth of ice locally at the protein-water interface or over a larger number of water layers near the protein. Recent THz studies [27] indicate that, at least for AFP in winter flounder, the effect appears to be delocalized.

The antifreeze activity of the AFP from the spruce budworm Choristoneura fumiferana [28], shown in Figure 1, can apparently be attributed in part to specific residues located on part of the surface of the protein. This protein is not a member of the Type I family. The protein structure contains three planes and mutation studies demonstrate that threonine-rich Plane 1 is the ice-binding plane. Mutation of just a few of the threonines to leucines (Figure 1) dramatically diminishes antifreeze activity [28]. Nutt and Smith [29] recently carried out MD simulations to examine the water dynamics in the hydration layer around the protein and found distinct dynamics around each of the three planes and noticeably slower dynamics around Plane 1. In this study we observe, like Nutt and Smith, quite distinct differences for the water dynamics around each of the three planes of the protein and in the hydrogen bond lifetimes for hydrogen bonds between the water molecules and the protein. Moreover, we examine a mutant that is antifreeze inactive and find that the mutation affects the hydrogen bond dynamics; that is, hydrogen bond lifetimes around the three planes are much closer to each other than in the wild type.

In the following section, we provide details of the computational methods and analysis. We then report results of our calculation of power spectra for water molecules near the three distinct planes of the protein and of our investigation of hydrogen bond lifetimes for bonds between water molecules and the protein. Concluding remarks are given in the final section.

\section{Computational Methods}

The initial coordinates of the antifreeze protein from the spruce budworm Choristoneura fumiferana were taken from the Protein Data Bank file 1L0S. Missing residues and hydrogen atoms were built into the structure and the iodated tyrosine Y26 required for the structure determination was reverted to a standard tyrosine using Swiss PDB Viewer [30]. For the mutant, four threonine residues on Plane 1 were mutated to leucines (Figure 1), a mutation that significantly reduces the antifreeze properties of the protein [17], to explore the effect of this mutation on the water dynamics and hydrogen bond lifetimes.

Both the wild-type and mutated structure were first minimized for 1000 steps with the steepest descent algorithm using the AMBER03 force field [31], after its solvation in a $70 \AA$ cubic water box of TIP5P water model. Then the systems, each of which contained 10539 water molecules, were equilibrated for $400 \mathrm{ps}$. For the first $100 \mathrm{ps}$ the positions of the proteins were restrained and in the latter $300 \mathrm{ps}$ they were released. Constraints were applied to all bonds to hydrogen with the SHAKE algorithm and periodic boundary conditions were applied. All the classical MD simulations were performed on the systems in canonical (NVT) ensemble with the GROMACS software package [32]. Following equilibration, trajectories of $2 \mathrm{~ns}$ were obtained at $300 \mathrm{~K}$ with a Nose-Hoover thermostat $[22,23]$. Nonbonded interactions were gradually brought to zero by a shift function for the electrostatics as well as a switch function for van der Waals interactions between 10 and $12 \AA[24,25]$. All the simulations were performed by integrating Newton's equations of motion with the Verlet algorithm [26] using 1 fs time steps. The system coordinates and velocities were stored every $5 \mathrm{fs}$, and the velocity autocorrelation function (VACF) was averaged over $15 \mathrm{ps}$ time segments of the trajectory for the oxygen atoms that survive in the first hydration shell of thickness $5 \AA$ as well as for those that hydrogen bond to the proteins. (Criteria for hydrogen bonds are specified below.) The VACF is defined as

$$
C_{V}(t)=\frac{\left\langle\mathbf{v}_{i}(t) \cdot \mathbf{v}_{i}(0)\right\rangle}{\left\langle\mathbf{v}_{i}(0) \cdot \mathbf{v}_{i}(0)\right\rangle},
$$

where $\mathbf{v}_{i}(t)$ is the velocity vector of the oxygen atom at time, $t$. The angular brackets denote averaging over all atoms of the particular type present in the hydration shell and over different reference initial times. Power spectra were obtained by Fourier transform of $C_{V}(t)$. The power spectra correspond to the vibrational density of the water. The vibrational density of protein molecules has been discussed elsewhere [11,33-36].

Hydrogen bond time correlation functions, $C_{\mathrm{HB}}(t)$, were also computed for bonds between water molecules and the protein at $300 \mathrm{~K} . C_{\mathrm{HB}}(t)$ is defined as the probability that, if a hydrogen bond between donor, $\mathrm{D}$, and acceptor, $\mathrm{A}$, exists at $t=0$, then it still exists at time, $t$, even if the bond broke at some intermediate time [37]. We adopt a standard criterion for hydrogen bonds, that is, a DA distance of $3.5 \AA$ and a D$\mathrm{H}-\mathrm{A}$ angle greater than $150^{\circ}[1,38,39]$.

\section{Results and Discussion}

3.1. VACF Power Spectra. A protein molecule perturbs the regular water-water hydrogen bond network in bulk water 


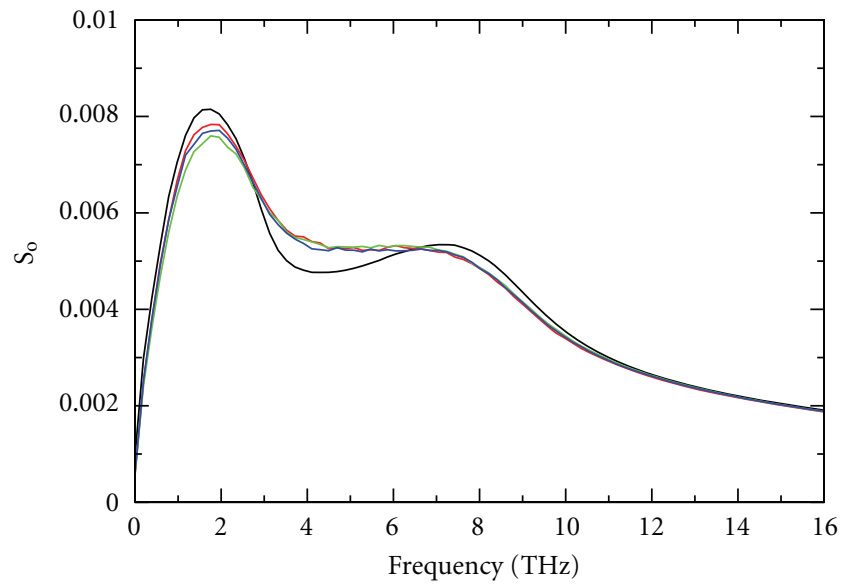

(a)

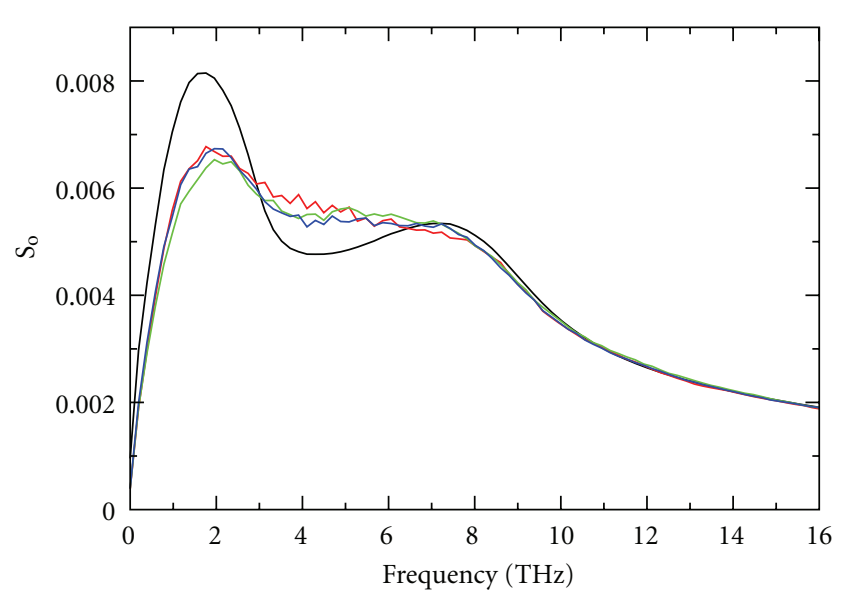

(b)

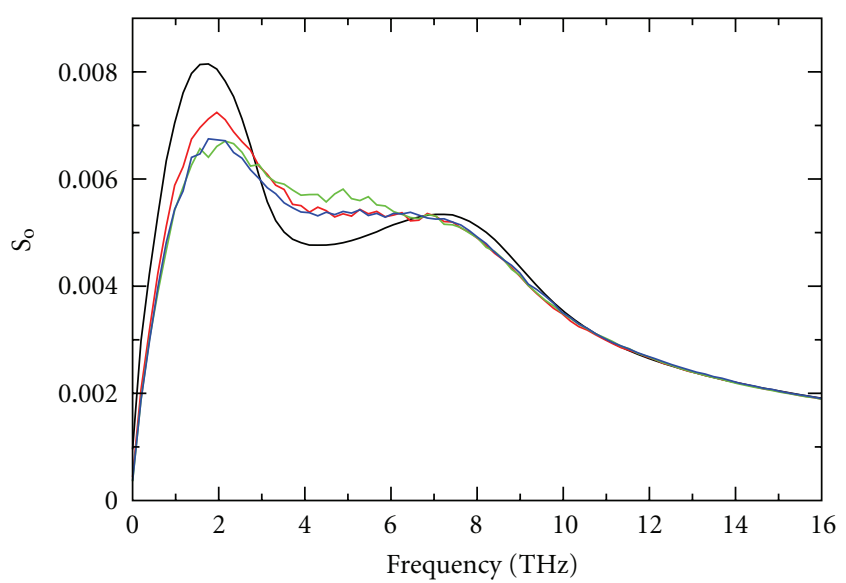

(c)

FIGURE 2: Power spectra of the velocity autocorrelation function of water in hydration layers around Plane 1 (red), Plane 2 (green), and Plane 3 (blue) of the protein, as well as for bulk water (black), at $300 \mathrm{~K}$. (a) Power spectrum of water in the hydration layer, taken to be $5 \AA$ from the surface of the wild-type AFP. (b) Power spectrum of water hydrogen bonding to wild type AFP. (c) Same as (b), but for mutant AFP.

with the formation of protein-water hydrogen bonds and influences the water dynamics in the hydration layer around the protein surface. We have calculated at $300 \mathrm{~K}$ the velocity autocorrelation function and its power spectra for the water molecules in the hydration layer around the protein, which can provide insights into $\mathrm{THz}$ spectra of solvated proteins [40]. We have carried out this calculation both for the water molecules that form hydrogen bonds with the amino acid residues of the three planes of the protein and for the larger number of water molecules within a layer of thickness $5 \AA$ from the protein [41]. Power spectra are plotted in Figure 2 for the wild-type and mutant at $300 \mathrm{~K}$. The results of a separate MD simulation of pure TIP5P water under the same conditions are also included for comparison.

We consider first the power spectra for bulk water, which appears in each of the panels in Figure 2. We observe two bands in the power spectra of water at about 2 and $8 \mathrm{THz}$. The lower frequency band has been interpreted [42, 43 ] as corresponding to the $\mathrm{O} \cdots \mathrm{O} \cdots \mathrm{O}$ bending mode from triplets of hydrogen-bonded water molecules and the higher frequency band as $\mathrm{O} \cdots \mathrm{O}$ stretching mode between pairs of hydrogen-bonded water molecules. Turning to the hydration water, the results plotted in Figure 2(a) reveal a clear blue shift in $S_{O}(\omega)$ for the band corresponding to the $\mathrm{O} \cdots \mathrm{O} \cdots \mathrm{O}$ bending for water. The shift is very similar for the water molecules in the $5 \AA$ hydration layer around each of the three planes. A blue shift in the same spectral region has been observed for water molecules in the hydration layer around helices of the villin headpiece subdomain, HP-36 [44]. Figure 2(b) gives the result for the hydration layer around the wild-type AFP, and we observe similar results for the mutant (not shown). Overall, we find that for the water molecules in the $5 \AA$ hydration layer around the protein there is little difference among the spectra obtained for the water near Planes 1, 2, or 3.

For the water molecules hydrogen bonded to the protein we observe distinct differences in the power spectra of the velocity autocorrelation function for each of the planes. The power spectra for the water hydrogen bonded to the protein exhibit again peaks near 2 and $8 \mathrm{THz}$, but the intensity of 


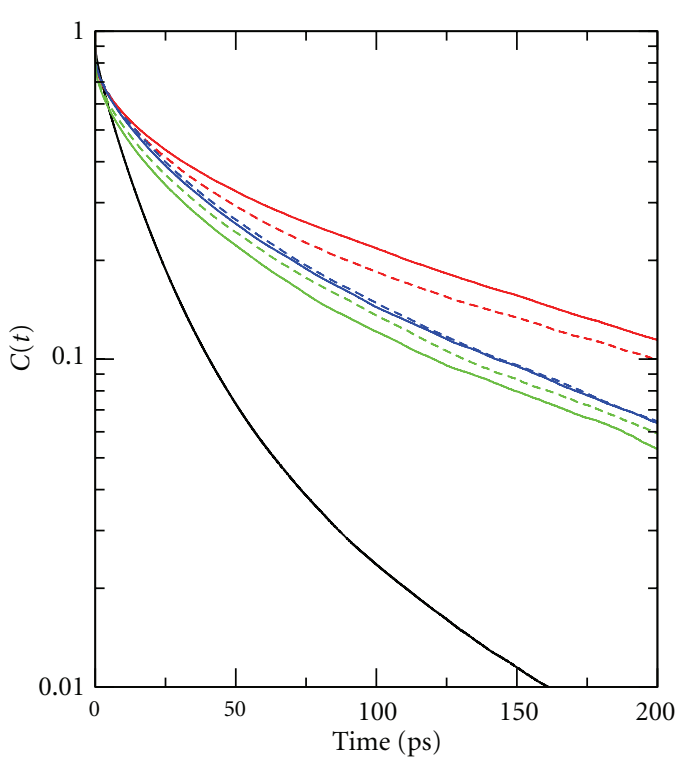

FIgURE 3: The hydrogen-bond time correlation function plotted for water hydrogen bonded to Plane 1 (red), Plane 2 (blue), and Plane 3 (green) of wild-type (solid) and mutant (dotted) AFP at $300 \mathrm{~K}$. The result for hydrogen bonds between water molecules in the bulk is plotted (black) for comparison.

the $2 \mathrm{THz}$ peak is smaller than for the hydration water, and the peak corresponding to the $\mathrm{O} \cdots \mathrm{O} \cdots \mathrm{O}$ bending appears even further broadened and blue shifted compared to bulk water than the peak for the hydration water in the $5 \AA$ layer around the protein. This could be related to the more restricted dynamics of the water molecules hydrogen bonded to the protein. Indeed, we have computed the power spectrum for bulk water at $250 \mathrm{~K}$ and for water molecules in the hydration shell and found the first peak for bulk water at this lower temperature to have a smaller intensity, and similar to that for the hydration water [45]. For the wild type we observe that Plane 1 exhibits a greater intensity on the blue edge of the lower frequency band compared to the other planes, whereas for the mutant the intensity is also greater but on the red side of the band. Because the power spectra for the wild type and the mutant are distinct, we expect that differences in the $\mathrm{THz}$ spectra of the wild type and the mutant can be detected.

3.2. Hydrogen Bond Correlation Function. We plot in Figure 3 results for the hydrogen bond correlation function, $C_{\mathrm{HB}}(t)$, defined in Section 2, to times of 200 ps for hydrogen bonds between water molecules and protein atoms on Planes 1,2 , and 3 of the wild type and mutant, as well as between water molecules in the bulk for comparison. All the simulations were carried out at $300 \mathrm{~K}$. Overall, the observed slow rearrangement times for hydrogen bonds between water molecules and the protein compared to hydrogen bonds between water molecules in the bulk are consistent with expectations for water molecules in the hydration layer around a protein [46, 47]. Nutt and Smith [29] computed the hydrogen bond correlation function for bonds between water and the three planes of the wild type, and we focus here mainly on comparison of the wild-type results with the results for the mutant. The hydrogen bond lifetime for bonds between water and the protein survive longer than hydrogen bonds between water molecules in the bulk, as seen in numerous previous simulation studies [1, 9, 38, 48, 49]. However, we also observe differences for hydrogen bonds between water and atoms on different planes of the protein. Considering first the wild type, we find, as did Nutt and Smith [29], that $C_{\mathrm{HB}}(t)$ for hydrogen bonds between water molecules and atoms of Plane 1 decays significantly slower than $C_{\mathrm{HB}}(t)$ for bonds between water molecules and the other two planes.

Interestingly, we find the hydrogen bond correlation functions for hydrogen bonds between water and the three protein planes to be noticeably closer to each other for the mutant than for the wild type out to the 100 picosecond time scale. The antifreeze activity of the protein decreases dramatically when replacing four of the threonines on Plane 1, indicated in Figure 1, with leucines [28] and we observe in our MD simulations that the hydrogen bond lifetimes for bonds between water molecules and atoms of each plane become similar to one another with this mutation. Only four point mutations have a sizable effect on the hydrogen bond dynamics, indicating the effect may not simply be local, but may influence the orientation of many water molecules. Such a nonlocal effect on the orientation of hydration waters by point mutation has been illustrated recently for a simple protein-sized model system [50].

\section{Concluding Remarks}

In this work, we examined the power spectrum of the velocity autocorrelation function for water molecules near the surface of the antifreeze protein (AFP) from the spruce budworm Choristoneura fumiferana and analyzed the hydrogen bond lifetimes for bonds between water molecules and the protein. We explored effects of the heterogeneity of the protein surface, in particular the distinctive properties of the water and protein-water interactions on the three planes of the protein, one of which is vital to the function of this AFP, and how the dynamics is affected by mutation.

For the power spectra of the water in the hydration layer of the AFP and the subset of that water that hydrogen bonds to the protein we find a blue shift of the roughly $2 \mathrm{THz}$ band compared to the same band in bulk water with a more pronounced shift for the water molecules that are hydrogen bonded to the protein residues. Although the power spectra for the water molecules within $5 \AA$ of each of the planes of the protein appear quite similar, power spectra for the water molecules hydrogen bonded to different planes of the protein exhibit distinct spectra in the range $1-4 \mathrm{THz}$. The differences among the power spectra for the water molecules hydrogen bonding to each of the three planes are influenced by mutation. We expect that $\mathrm{THz}$ measurements, which are highly sensitive to the hydration water [48, 51-55], will reveal differences between the wild type and mutant. Recent $\mathrm{THz}$ experiments [49] on a $\lambda$-repressor fragment indicate that 
only a few point mutations can give rise to very different $\mathrm{THz}$ spectra.

The hydrogen bond time correlation function was computed for hydrogen bonds between water molecules and each of the planes of the protein. For wild type AFP we observe differences in the hydrogen bond lifetimes for bonds between water and the three planes. The longest lifetimes are found for hydrogen bonds between water molecules and Plane 1, the ice-binding plane of the protein, consistent with results of previous simulations [29]. We observe that by introducing only four mutations to Plane 1, mutations that have been observed to substantially diminish the AFP activity of the protein [28], the hydrogen bond correlation function for bonds between water molecules and each of the three planes are similar to one another. Overall, mutation is seen to modify hydrogen bonding over a wide range of time scales observable both in the power spectra and analysis of hydrogen bond lifetimes. These measures of hydrogen bonding at the protein-water interface aid in quantifying the complexity and heterogeneity of the interactions between water and the antifreeze protein and reveal regions of the protein-water interface important for antifreeze activity.

\section{Acknowledgments}

Support from the National Science Foundation (NSF CHE0910669) and from the Volkswagen Foundation (VW Stiftung Az I/84 302) is gratefully acknowledged.

\section{References}

[1] D. J. Tobias, N. Sengupta, and M. Tarek, "Molecular dynamics simulation studies of coupled protein and water dynamics," in Proteins: Energy, Heat and Signal Flow, D. M. Leitner and J. E. Straub, Eds., pp. 361-386, Taylor \& Francis, Boca Raton, Fla, USA, 2009.

[2] M. E. Johnson, C. Malardier-Jugroot, R. K. Murarka, and T. Head-Gordon, "Hydration water dynamics near biological interfaces," Journal of Physical Chemistry B, vol. 113, no. 13, pp. 4082-4092, 2009.

[3] A. R. Bizzarri and S. Cannistraro, "Molecular dynamics of water at the protein-solvent interface," Journal of Physical Chemistry B, vol. 106, no. 26, pp. 6617-6633, 2002.

[4] P. J. Steinbach and B. R. Brooks, "Protein hydration elucidated by molecular dynamics simulation," Proceedings of the National Academy of Sciences of the United States of America, vol. 90, no. 19, pp. 9135-9139, 1993.

[5] D. N. LeBard and D. V. Matyushov, "Ferroelectric hydration shells around proteins: electrostatics of the protein-water interface," Journal of Physical Chemistry B, vol. 114, no. 28, pp. 9246-9258, 2010.

[6] X. Yu, J. Park, and D. M. Leitner, "Thermodynamics of protein hydration computed by molecular dynamics and normal modes," Journal of Physical Chemistry B, vol. 107, no. 46, pp. 12820-12828, 2003.

[7] F. Despa, A. Fernández, and R. S. Berry, "Publisher's notedielectric modulation of biological water," Physical Review Letters, vol. 93, no. 26, Article ID 228104, 1 pages, 2004.

[8] R. Gnanasekaran, J. K. Agbo, and D. M. Leitner, "Communication maps computed for homodimeric hemoglobin: computational study of water-mediated energy transport in proteins," Journal of Chemical Physics, vol. 135, no. 6, Article ID 065103, 10 pages, 2011.

[9] R. Gnanasekaran, Y. Xu, and D. M. Leitner, "Dynamics of water clusters confined in proteins: a molecular dynamics simulation study of interfacial waters in a dimeric hemoglobin," Journal of Physical Chemistry B, vol. 114, no. 50, pp. 1698916996, 2010.

[10] A. Lervik, F. Bresme, S. Kjelstrup, D. Bedeaux, and J. M. Rubi, "Heat transfer in protein-water interfaces," Physical Chemistry Chemical Physics, vol. 12, no. 7, pp. 1610-1617, 2010.

[11] D. M. Leitner, M. Havenith, and M. Gruebele, "Biomolecule large-amplitude motion and solvation dynamics: modelling and probes from $\mathrm{THz}$ to X-rays," International Reviews in Physical Chemistry, vol. 25, no. 4, pp. 553-582, 2006.

[12] L. Mitra, N. Smolin, R. Ravindra, C. Royer, and R. Winter, "Pressure perturbation calorimetric studies of the solvation properties and the thermal unfolding of proteins in solutionexperiments and theoretical interpretation," Physical Chemistry Chemical Physics, vol. 8, no. 11, pp. 1249-1265, 2006.

[13] S. K. Pal, J. Peon, and A. H. Zewail, "Biological water at the protein surface: dynamical solvation probed directly with femtosecond resolution," Proceedings of the National Academy of Sciences of the United States of America, vol. 99, no. 4, pp. 1763-1768, 2002.

[14] W. Doster and M. Settles, "The dynamical transition in proteins: the role of hydrogen bonds," in Hydration Processes in Biology: Experimental and Theoretical Approaches, M.-C. Bellissent-Funel, Ed., pp. 177-195, IOS Press, Amsterdam, The Netherlands, 1999.

[15] E. Persson and B. Halle, "Cell water dynamics on multiple time scales," Proceedings of the National Academy of Sciences of the United States of America, vol. 105, no. 17, pp. 6266-6271, 2008.

[16] H. Frauenfelder, P. W. Fenimore, G. Chen, and B. H. McMahon, "Protein folding is slaved to solvent motions," Proceedings of the National Academy of Sciences of the United States of America, vol. 103, no. 42, pp. 15469-15472, 2006.

[17] S. P. Graether, Biochemistry and Function of Antifreeze Proteins, Nova Science, New York, NY, USA, 2011.

[18] J. G. Duman, K. R. Walters, T. Sformo et al., "Antifreeze and ice-nucleator proteins," in Low Temperature Biology of Insects, D. L. Delinger and R. E. Lee, Eds., pp. 59-90, Cambridge University Press, New York, NY, USA, 2010.

[19] B. Moffatt, V. Ewart, and A. Eastman, "Cold comfort: plant antifreeze proteins," Physiologia Plantarum, vol. 126, no. 1, pp. 5-16, 2006.

[20] L. Pham, R. Dahiya, and B. Rubinsky, "An in vivo study of antifreeze protein adjuvant cryosurgery," Cryobiology, vol. 38, no. 2, pp. 169-175, 1999.

[21] A. L. DeVries and D. E. Wohlschlag, "Freezing resistance in some antarctic fishes," Science, vol. 163, no. 3871, pp. 10731075, 1969.

[22] J. A. Raymond and A. L. DeVries, "Adsorption inhibition as a mechanism of freezing resistance in polar fishes," Proceedings of the National Academy of Sciences of the United States of America, vol. 74, no. 6, pp. 2589-2593, 1977.

[23] J. A. Raymond, P. W. Wilson, and A. L. DeVries, "Inhibition of growth of nonbasal planes in ice by fish antifreezes," Proceedings of the National Academy of Sciences of the United States of America, vol. 86, no. 3, pp. 881-885, 1989.

[24] C. A. Knight, C. C. Cheng, and A. L. DeVries, "Adsorption of $\alpha$-helical antifreeze peptides on specific ice crystal surface planes," Biophysical Journal, vol. 59, no. 2, pp. 409-418, 1991. 
[25] J. Duman and A. L. DeVries, "Isolation, characterization, and physical properties of protein antifreezes from the winter flounder, pseudopleuronectes americanus," Comparative Biochemistry and Physiology, vol. 54, no. 3, pp. 375-380, 1976.

[26] A. D. Haymet, L. G. Ward, M. M. Harding, and C. A. Knight, "Valine substituted winter flounder "antifreeze": preservation of ice growth hysteresis," FEBS Letters, vol. 430, no. 3, pp. 301306, 1998.

[27] S. Ebbinghaus, K. Meister, B. Born, A. L. Devries, M. Gruebele, and M. Havenith, "Antifreeze glycoprotein activity correlates with long-range protein-water dynamics," Journal of the American Chemical Society, vol. 132, no. 35, pp. 12210$12211,2010$.

[28] S. P. Graether, M. J. Kuiper, and S. M. Gagne, "Beta-helix structure and ice-binding properties of a hyperactive antifreeze protein from an insect," Nature, pp. 325-328, 2000.

[29] D. R. Nutt and J. C. Smith, "Dual function of the hydration layer around an antifreeze protein revealed by atomistic molecular dynamics simulations," Journal of the American Chemical Society, vol. 130, no. 39, pp. 13066-13073, 2008.

[30] N. Guex and M. C. Peitsch, "SWISS-MODEL and the SwissPdbViewer: an environment for comparative protein modeling," Electrophoresis, vol. 18, no. 15, pp. 2714-2723, 1997.

[31] Y. Duan, C. Wu, S. Chowdhury et al., "A point-charge force field for molecular mechanics simulations of proteins based on condensed-phase quantum mechanical calculations," Journal of Computational Chemistry, vol. 24, no. 16, pp. 1999-2012, 2003.

[32] H. J. C. Berendsen, D. Spoel, and R. V. Drunen, "GROMACS: a message-passing parallel molecular dynamics implementation," Computer Physics Communications, vol. 91, no. 1-3, pp. 43-56, 1995.

[33] X. Yu and D. M. Leitner, "Vibrational energy transfer and heat conduction in a protein," Journal of Physical Chemistry B, vol. 107, no. 7, pp. 1698-1707, 2003.

[34] X. Yu and D. M. Leitner, "Anomalous diffusion of vibrational energy in proteins," Journal of Chemical Physics, vol. 119, no. 23, pp. 12673-12679, 2003.

[35] X. Yu and D. M. Leitner, "Heat flow in proteins: computation of thermal transport coefficients," Journal of Chemical Physics, vol. 122, no. 5, Article ID 054902, 11 pages, 2005.

[36] D. M. Leitner, "Vibrational energy transfer and heat conduction in a one-dimensional glass," Physical Review B, vol. 64, no. 9, Article ID 094201, 9 pages, 2001.

[37] B. Bagchi, "Water dynamics in the hydration layer around proteins and micelles," Chemical Reviews, vol. 105, no. 9, pp. 3197-3219, 2005.

[38] M. Tarek and D. J. Tobias, "Role of protein-water hydrogen bond dynamics in the protein dynamical transition," Physical Review Letters, vol. 88, no. 13, Article ID 138101, 4 pages, 2002.

[39] A. Luzar and D. Chandler, "Hydrogen-bond kinetics in liquid water," Nature, vol. 379, no. 6560, pp. 55-57, 1996.

[40] M. Heyden and M. Havenith, "Combining THz spectroscopy and MD simulations to study protein-hydration coupling," Methods, vol. 52, no. 1, pp. 74-83, 2010.

[41] S. Bandyopadhyay, S. Chakraborty, and B. Bagchi, "Secondary structure sensitivity of hydrogen bond lifetime dynamics in the protein hydration layer," Journal of the American Chemical Society, vol. 127, no. 47, pp. 16660-16667, 2005.

[42] G. E. Walrafen and Y. C. Chu, "Linearity between structural correlation length and correlated-proton Raman intensity from amorphous ice and supercooled water up to dense supercritical steam," Journal of Physical Chemistry, vol. 99, no. 28, pp. 11225-11229, 1995.
[43] G. E. Walrafen, Y. C. Chu, and G. J. Piermarini, "Low-frequency Raman scattering from water at high pressures and high temperatures," Journal of Physical Chemistry, vol. 100, no. 24, pp. 10363-10372, 1996.

[44] S. Chakraborty, S. K. Sinha, and S. Bandyopadhyay, "Lowfrequency vibrational spectrum of water in the hydration layer of a protein: a molecular dynamics simulation study," Journal of Physical Chemistry B, vol. 111, no. 48, pp. 13626-13631, 2007.

[45] Y. Xu, R. Gnanasekaran, and D. M. Leitner, (published results).

[46] N. Nandi and B. Bagchi, "Dielectric relaxation of biological water," Journal of Physical Chemistry B, vol. 101, no. 50, pp. 10954-10961, 1997.

[47] S. K. Pal, J. Peon, B. Bagchi, and A. H. Zewail, "Biological water: femtosecond dynamics of macromolecular hydration," Journal of Physical Chemistry B, vol. 106, no. 48, pp. 1237612395, 2002.

[48] S. Ebbinghaus, S. J. Kim, M. Heyden et al., "An extended dynamical hydration shell around proteins," Proceedings of the National Academy of Sciences of the United States of America, vol. 104, no. 52, pp. 20749-20752, 2007.

[49] S. Ebbinghaus, S. J. Kim, M. Heyden et al., "Protein sequence- and $\mathrm{pH}$-dependent hydration probed by terahertz spectroscopy," Journal of the American Chemical Society, vol. 130, no. 8, pp. 2374-2375, 2008.

[50] A. D. Friesen and D. V. Matyushov, "Non-Gaussian statistics of electrostatic fluctuations of hydration shells," Journal of Chemical Physics, vol. 135, no. 10, Article ID 104501, 7 pages, 2011.

[51] B. Born, S. J. Kim, S. Ebbinghaus, M. Gruebele, and M. Havenith, "The terahertz dance of water with the proteins: the effect of protein flexibility on the dynamical hydration shell of ubiquitin," Faraday Discussions, vol. 141, pp. 161-173, 2008.

[52] U. Heugen, G. Schwaab, E. Bründermann et al., "Soluteinduced retardation of water dynamics probed directly by terahertz spectroscopy," Proceedings of the National Academy of Sciences of the United States of America, vol. 103, no. 33, pp. 12301-12306, 2006.

[53] M. Heyden, E. Bründermann, U. Heugen, G. Niehues, D. M. Leitner, and M. Havenith, "Long-range influence of carbohydrates on the solvation dynamics of water-answers from terahertz absorption measurements and molecular modeling simulations," Journal of the American Chemical Society, vol. 130, no. 17, pp. 5773-5779, 2008.

[54] J. Knab, J. Y. Chen, and A. G. Markelz, "Hydration dependence of conformational dielectric relaxation of lysozyme," Biophysical Journal, vol. 90, no. 7, pp. 2576-2581, 2006.

[55] N. Q. Vinh, S. J. Allen, and K. W. Plaxco, "Dielectric spectroscopy of proteins as a quantitative experimental test of computational models of their low-frequency harmonic motions," Journal of the American Chemical Society, vol. 133, no. 23, pp. 8942-8947, 2011. 

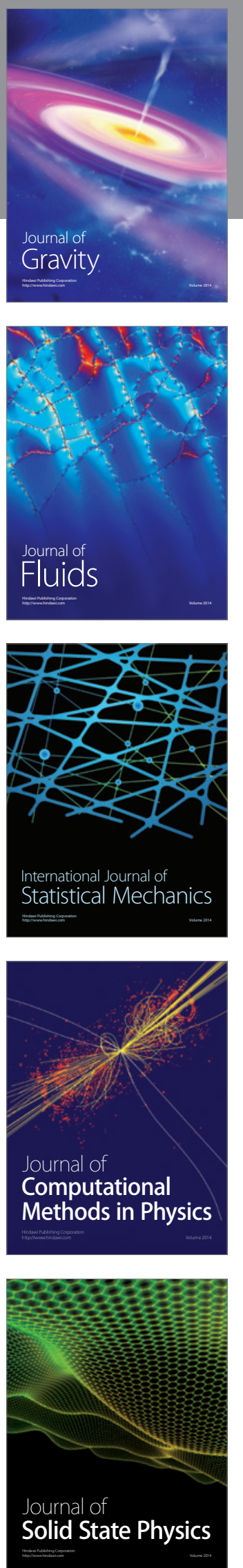

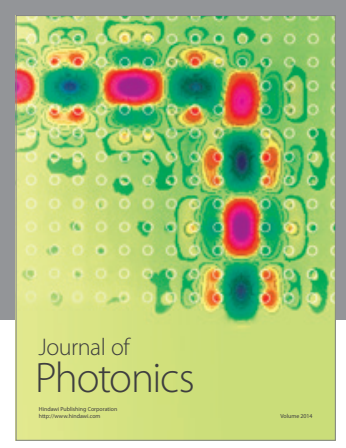

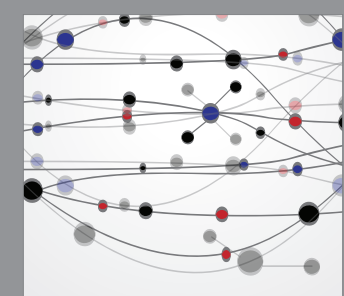

The Scientific World Journal
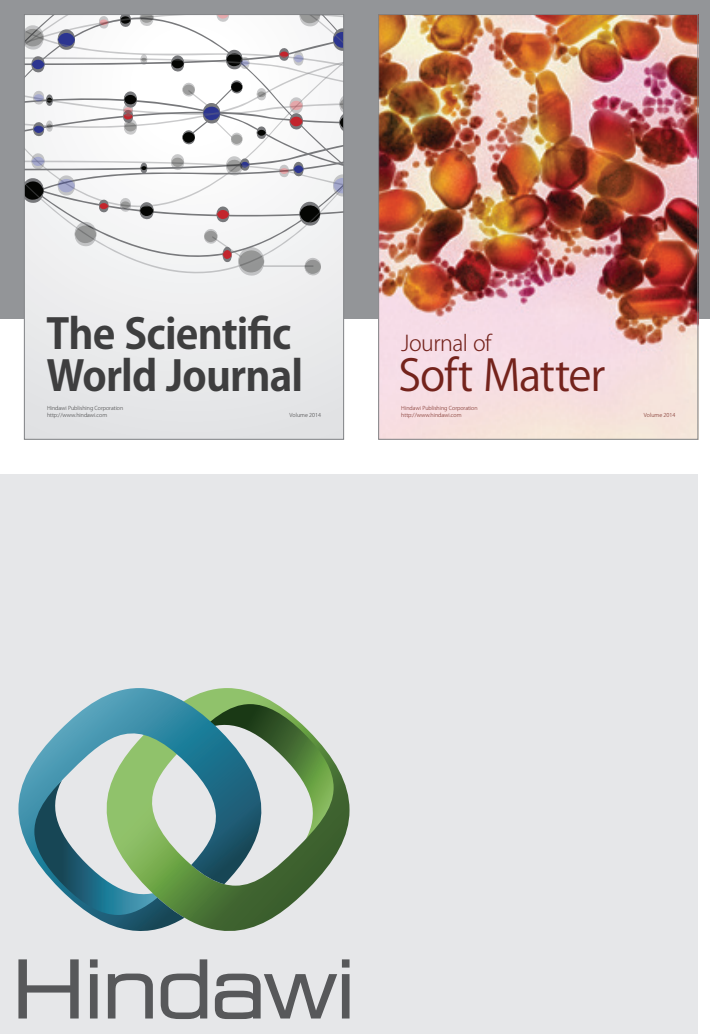

Submit your manuscripts at

http://www.hindawi.com
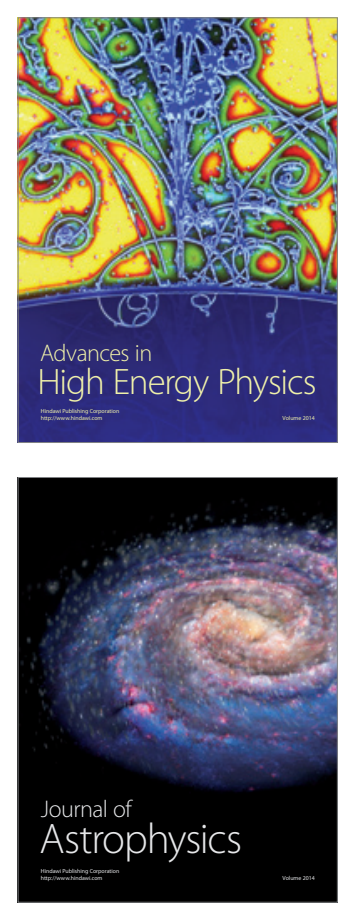
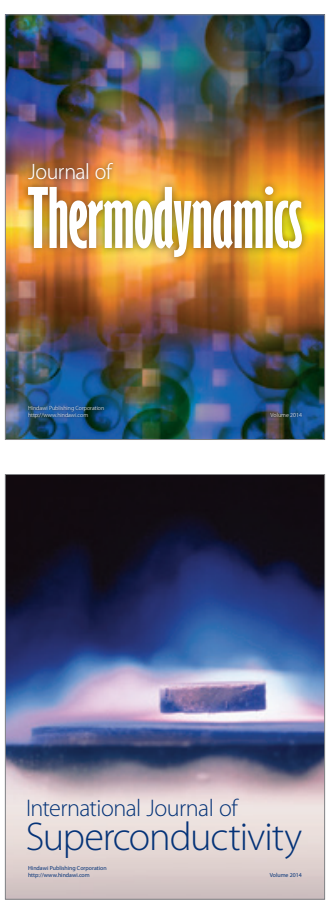
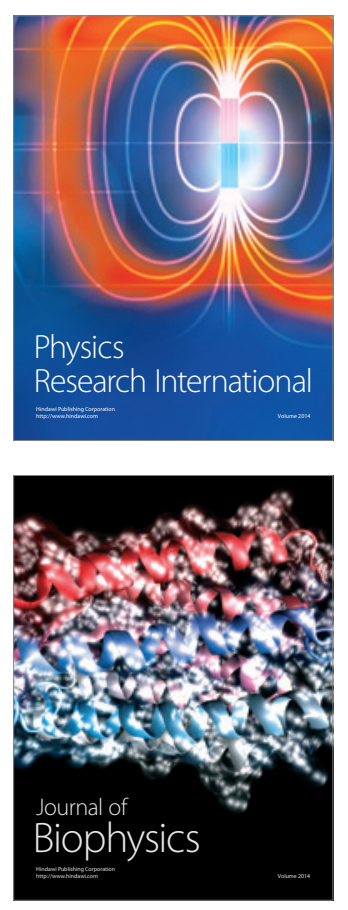
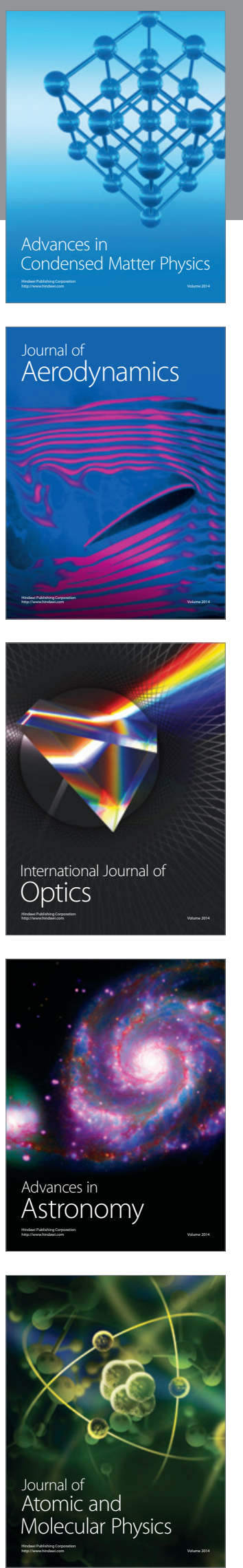